\title{
Energetics and Electronic Structures of Individual Atoms Adsorbed on Carbon Nanotubes
}

\author{
E. Durgun, S. Dag, S. Ciraci,* and O. Giilseren \\ Department of Physics, Bilkent University, Ankara 06800, Turkey \\ Received: June 29, 2003; In Final Form: September 30, 2003
}

\begin{abstract}
The adsorption of individual atoms on the semiconducting and metallic single-walled carbon nanotubes (SWNT) has been investigated by using the first principles pseudopotential plane wave method within density functional theory. The stable adsorption geometries and binding energies have been determined for a large number of foreign atoms ranging from alkali and simple metals to the transition metals and group IV elements. We have found that the character of the bonding and associated physical properties strongly depends on the type of adsorbed atoms, in particular, on their valence electron structure. Our results indicate that the properties of SWNTs can be modified by the adsorbed foreign atoms. Although the atoms of good conducting metals, such as $\mathrm{Zn}, \mathrm{Cu}, \mathrm{Ag}$, and $\mathrm{Au}$, form very weak bonds, transition-metal atoms such as $\mathrm{Ti}, \mathrm{Sc}, \mathrm{Nb}$, and $\mathrm{Ta}$ and group IV elements $\mathrm{C}$ and $\mathrm{Si}$ are adsorbed with a relatively high binding energy. Owing to the curvature effect, these binding energies are larger than the binding energies of the same atoms on the graphite surface. We have showed that the adatom carbon can form strong and directional bonds between two SWNTs. These connects can be used to produce nanotube networks or grids. Most of the adsorbed transition-metal atoms excluding $\mathrm{Ni}, \mathrm{Pd}$, and $\mathrm{Pt}$ have a magnetic ground state with a significant magnetic moment. Our results suggest that carbon nanotubes can be functionalized in different ways by their coverage with different atoms, showing interesting applications such as 1D nanomagnets or nanoconductors, conducting connects, and so forth.
\end{abstract}

\section{Introduction}

Single-walled carbon nanotubes ${ }^{1,2}$ (SWNT) can be functionalized by the adsorption of atoms or molecules, which can induce dramatic changes in the physical and chemical properties of the bare tube. The functionalization of SWNTs is of interest for two possible, insofar technologically important applications, namely, the fabrication of metallic nanowires and nanomagnets. Because nanoelectronics are promising rapid miniaturization providing higher and higher device density and operation speeds, the fabrication of interconnects with high conductance and low energy dissipation appears to be a real technological problem. Very thin metal wires and atomic chains were produced by retracting the STM tip from an indentation and then by thinning the neck of the materials that wet the tip. ${ }^{3-5}$ Although those nanowires produced so far played a crucial role in understanding the quantum effects in electronic and thermal conductance, ${ }^{6-10}$ they were neither stable nor reproducible to offer any relevant technological application. Nowadays, the most practical and realizable method to fabricate nanowires relies on carbon nanotubes. Earlier experimental studies have indicated that SWNTs can serve as templates to produce reproducible, very thin metallic wires with controllable sizes. ${ }^{11}$ These metallic nanowires can be used as conducting connects and hence are important in nanodevices based on molecular electronics. Recently, Zhang et al. ${ }^{12}$ have shown that continuous Ti coating of varying thickness and quasi-continuous coating of $\mathrm{Ni}$ and Pd can be obtained by using electron beam evaporation techniques. However, metal atoms such as $\mathrm{Au}, \mathrm{Al}, \mathrm{Fe}$, and $\mathrm{Pb}$ were able to form only isolated discrete particles or clusters instead of a continuous coating of SWNTs.

* Corresponding author. E-mail: ciraci@fen.bilkent.edu.tr.
Not only metallic connects but also the contacts of metal electrodes themselves are crucial to the operation of devices based on nanotubes. Low-resistance ohmic contacts to metallic and semiconducting SWNTs were achieved by Ti and Ni. ${ }^{13}$ The formation of a Schottky barrier at the contact has been found to be responsible for the operation of field-emission transistors made from SWNTs. ${ }^{14-16}$

Theoretical studies ${ }^{17}$ have indicated that stable rings and tubes of $\mathrm{Al}$ atoms can form around a semiconducting SWNT. It is argued that either persistent currents through these conducting nanorings or conversely very high magnetic fields can be induced at their center. ${ }^{17}$ Such a setup has been also proposed as possible qubits in quantum computation. ${ }^{18}$ It has been shown experimentally that the implementation of iron atoms inside the tube can give rise to magnetization. ${ }^{19}$ Such a system may be specified as a nanomagnet and can be used in several applications ranging from various research tools to high-density storage devices.

It is expected that novel molecular nanomagnets and electromagnetic devices can be generated from these metallic nanostructures formed by the adsorption of specific atoms on the surface of SWNTs. Thus, the study of the adsorption of atoms on nanotube surfaces is essential to achieving lowresistance ohmic contacts to nanotubes, to produce nanowires with controllable sizes, and to fabricate nanomagnets and functional nanodevices.

This paper presents an extensive study of the adsorption of individual atoms on the surface of a semiconducting $(8,0)$ and also a metallic $(6,6)$ SWNT. The binding geometry and binding energy and resulting electronic structure of various atoms (ranging from alkali and simple metals to group IV elements and including most of the transition metals) have been investigated. Results of our earlier studies on hydrogen and oxygen 
atoms, ${ }^{20,21}$ which are relevant to this paper, have been also included for the sake of completeness. Preliminary results of this extensive study were presented earlier as a short communication. ${ }^{22}$ Our prime objective is to reveal the character and geometry of the bonding and to understand why some metal atoms form strong bonds while others are only weakly bound. The effect of the adsorption on the physical properties, such as electronic and magnetic, is another issue that we deal with in detail in this work. Specifically, we addressed the question of whether the ground state of a SWNT with an adsorbed atom has a net spin. We have explored the situation of whether the magnetic ground state gives rise to bands with one type of spin. Finally, we discuss the subject of connecting the SWNTs and hence making networks by carbon adatoms. We believe that our results are important to applied and theoretical research such as the coating of carbon nanotubes, the design and fabrication of functionalized nanodevices and nanomagnets, spintronics, gluing SWNTs to generate cross, $\mathrm{T}$, and $\mathrm{Y}$ structures, and forming metal-SWNT junctions and contacts.

\section{Method of Calculations}

Our study deals with the adsorption of 27 different atoms on the $(8,0)$ zigzag SWNT and four atoms on the $(6,6)$ armchair SWNT. The atomic structure, binding geometry, binding energy, and resulting electronic structure of an individual-atom adsorbed SWNT have been calculated by using the first principles pseudopotential plane wave method within the generalized gradient approximation (GGA) ${ }^{23}$ Earlier, the first principles pseudopotential method using the supercell method had been demonstrated to provide accurate predictions of the mechanical and electronic properties of various zigzag and armchair SWNTs for undeformed and radially deformed cases. ${ }^{20,24,25}$

Spin-unpolarized and spin-polarized (relaxed) calculations have been carried out for a single atom, a bare SWNT, and a single-atom absorbed SWNT. Ultrasoft pseudopotentials ${ }^{26}$ and plane waves up to an energy cutoff of $300 \mathrm{eV}$ depending on the pseudopotential are used. The Brillouin zone of the supercell is sampled by $(1,1,11) k$ points within the Monkhorst-Pack special $k$-point scheme. ${ }^{27}$ Calculations have been performed in momentum space by using a periodically repeating tetragonal supercell with lattice constants $a_{\mathrm{s}}=b_{\mathrm{s}} \approx 15 \AA$ and $c_{\mathrm{s}}$. To minimize the adsorbate-adsorbate interaction, the lattice constant along the axis of the tube, $c_{\mathrm{s}}$, is taken to be twice the 1D lattice parameter of the bare tube (i.e., $c_{\mathrm{s}} \approx 2 c$ for the zigzag $\mathrm{SWNT}$, but $c_{\mathrm{s}} \approx 4 c$ for the armchair SWNT).

For the adsorption of individual atoms, we considered four possible sites (i.e., the $\mathrm{H}$ site above the hexagon, the $\mathrm{Z}$ and $\mathrm{A}$ sites above the zigzag and axial $\mathrm{C}-\mathrm{C}$ bonds, and the $\mathrm{T}$ site above the carbon atom) as described in Figure 1. The binding sites are determined by optimizing all atomic positions (adsorbate atom and 64 carbon atoms of the $(8,0)$ SWNT or 96 carbon atoms of the $(6,6)$ SWNT as well as $c_{\mathrm{S}}$ (hence $c$ ) using the conjugate gradient (CG) method). Binding energies are obtained from the expression

$$
E_{\mathrm{b}}^{\mathrm{u}(\mathrm{p})}=E_{\mathrm{T}}^{\mathrm{u}(\mathrm{p})}[\mathrm{SWNT}]+E_{\mathrm{T}}^{\mathrm{u}(\mathrm{p})}[\mathrm{A}]-E_{\mathrm{T}}^{\mathrm{u}(\mathrm{p})}[\mathrm{A}+\mathrm{SWNT}]
$$

in terms of the total energies corresponding to the fully optimized structure of the bare nanotube $\left(E_{\mathrm{T}}^{\mathrm{u}(\mathrm{p})}[\mathrm{SWNT}]\right)$, free atom $\mathrm{A}\left(E_{\mathrm{T}}^{\mathrm{u}(\mathrm{p})}[\mathrm{A}]\right)$, and atom $\mathrm{A}$ adsorbed on a $\operatorname{SWNT}\left(E_{\mathrm{T}}^{\mathrm{u}(\mathrm{p})}[\mathrm{A}\right.$ $+\mathrm{SWNT}])$. The superscript $\mathrm{u}(\mathrm{p})$ indicates spin-unpolarized (spin-polarized) energies. The binding energies $E_{\mathrm{b}}^{\mathrm{u}(\mathrm{p})}$ are obtained from the total energies corresponding to either the nonmagnetic (spin-unpolarized) state with zero net spin or the

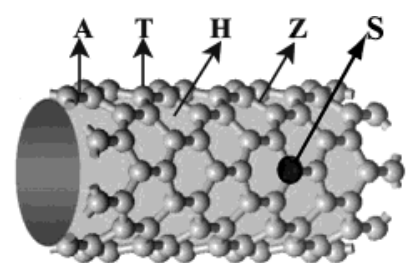

$(8,0)$ Zigzag SWNT

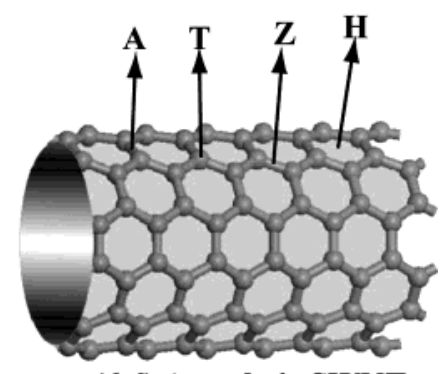

$(6,6)$ Armchair SWNT

Figure 1. Schematic description of different binding sites of individual atoms adsorbed on a zigzag $(8,0)$ tube. $\mathrm{H}$ : hollow; A: axial; Z: zigzag; T: top; S: substitution sites.

magnetic (spin-relaxed) state with net spin. A bare nanotube has a nonmagnetic ground state with zero net $\operatorname{spin} . E_{\mathrm{b}}^{\mathrm{u}(\mathrm{p})}>0$ corresponds to a CG-optimized stable structure and indicates the bonding (a local or global minimum on the BornOppenheimer surface). Only the short-range (chemical) interactions are included in the binding energy, $E_{\mathrm{b}}^{\mathrm{u}(\mathrm{p})}$. The longrange van der Waals interaction, $E_{\mathrm{vdW}}$, is expected to be much smaller than the chemisorption binding energy and is omitted. However, for specific elements such as $\mathrm{Mg}$ and $\mathrm{Zn}$, the binding energy is small, and the character of the bond is between chemisorption and physisorption. In this case, the weak and attractive van der Waals interaction energy is comparable with the physisorption energy. It can be calculated from the asymptotic form of the Lifshitz equation ${ }^{28}$

$$
E_{\mathrm{vdW}}=\sum_{i j} \frac{C_{6 i j}}{r_{i j}^{6}}
$$

with coefficients $C_{6 i j}$ obtained from the Slater-Kirkwood approximation. ${ }^{29}$ We note that the asymptotic form of $E_{\mathrm{vdW}}$ may not be accurate when the adatom-SWNT distance is small.

\section{Binding Geometries and Energies}

The cohesive energies of $\mathrm{C}$ atoms in the $(8,0)$ and $(6,6)$ tube (i.e., $E_{\mathrm{c}}=\left(N E_{\mathrm{T}}^{\mathrm{u}}[\mathrm{C}]-E_{\mathrm{T}}^{\mathrm{u}}[\mathrm{SWNT}]\right) / N$, with $N$ being the number of $\mathrm{C}$ atoms in the unit cell of the SWNT) are calculated to be $E_{\mathrm{c}}{ }^{(8,0)}=9.06 \mathrm{eV}$ and $E_{\mathrm{c}}{ }^{(6,6)}=9.14 \mathrm{eV}$, respectively. The optimized radii of the $(8,0)$ and $(6,6)$ SWNTs, $R^{(8,0)}=3.2 \AA$ and $R^{(6,6)}=4.1 \AA$, indicate that the $(8,0)$ tube has higher curvature. The zigzag $(8,0)$ SWNT is an insulator with a calculated band gap of $E_{\mathrm{g}}=0.64 \mathrm{eV}$. The $(6,6)$ armchair SWNT is a metal because $\pi^{*}$-conduction and $\pi$-valence bands cross at the Fermi level. As far as the electronic properties are concerned, our study has sampled two extreme cases in the class of SWNTs. The binding geometries and binding energies $E_{\mathrm{b}}^{\mathrm{u}}$ calculated from spin-unpolarized total energies as eq 1 are given in Table 1 for the $(8,0)$ SWNT and in Table 2 for the $(6,6)$ SWNT.

The interaction between SWNTs and most of the adatoms considered in this study is significant and results in the chemisorption bond. Thus, the binding energy corresponding 
TABLE 1: Calculated Binding Energies and Average Carbon-Adatom Bond Distances, $\bar{d}_{\mathrm{C}-\mathrm{A}}$ of Individual Atoms Adsorbed at $\mathrm{H}, \mathrm{Z}, \mathrm{A}$, and T Sites of the $(8,0)$ SWNT as Described in Figure $\mathbf{1}^{a}$

\begin{tabular}{llllllll}
\hline atom & \multicolumn{1}{c}{$\mathrm{H}(\mathrm{eV})$} & $\mathrm{A}(\mathrm{eV})$ & $\mathrm{Z}(\mathrm{eV})$ & $\mathrm{T}(\mathrm{eV})$ & $\Delta E_{\mathrm{T}}(\mathrm{eV})$ & $\bar{d}_{\mathrm{C}-\mathrm{A}}(\AA)$ & $\mu\left(\mu_{\mathrm{B}}\right)$ \\
\hline $\mathrm{Na}$ & 1.3 & 1.1 & 1.1 & 1.1 & & 2.3 & \\
$\mathrm{Mg}$ & 0.08 & 0.07 & 0.05 & 0.07 & & $3.8-$ & \\
$\mathrm{Sc}$ & $2.1(1.9)$ & 1.4 & 1.5 & $\rightarrow \mathrm{H}$ & 0.15 & $2.3(2.2)$ & $0.64(1)$ \\
$\mathrm{Ti}$ & $2.9(2.2)$ & 2.1 & 2.7 & 2.1 & 0.58 & $2.2(2.2)$ & $2.21(4)$ \\
$\mathrm{V}$ & $3.2(1.4)$ & 2.2 & $\rightarrow \mathrm{H}$ & $\rightarrow \mathrm{H}$ & 1.20 & $2.1(2.2)$ & $3.67(5)$ \\
$\mathrm{Cr}$ & $3.7(0.4)$ & 2.5 & $\rightarrow \mathrm{H}$ & $\rightarrow \mathrm{H}$ & 2.25 & $2.0(2.3)$ & $5.17(6)$ \\
$\mathrm{Mn}$ & $3.4(0.4)$ & 2.5 & $\rightarrow \mathrm{H}$ & $\rightarrow \mathrm{H}$ & 2.42 & $2.1(2.4)$ & $5.49(5)$ \\
$\mathrm{Fe}$ & $3.0(0.8)$ & 2.5 & $\rightarrow \mathrm{H}$ & 1.6 & 1.14 & $2.1(2.3)$ & $2.27(4)$ \\
$\mathrm{Co}$ & $2.8(1.7)$ & 2.5 & $\rightarrow \mathrm{H}$ & $\rightarrow \mathrm{H}$ & 0.41 & $2.1(2.0)$ & $1.05(3)$ \\
$\mathrm{Ni}$ & 2.2 & $2.4(1.7)$ & 2.3 & $\rightarrow \mathrm{A}$ & 0.02 & $1.9(1.9)$ & $0.04(2)$ \\
$\mathrm{Cu}$ & 0.5 & $0.8(0.7)$ & 0.6 & $\rightarrow \mathrm{A}$ & 0.03 & $2.1(2.1)$ & $0.53(1)$ \\
$\mathrm{Zn}$ & $0.05(0.04)$ & 0.05 & 0.03 & 0.04 & 0 & $3.7(3.7)$ & $0(0)$ \\
$\mathrm{Nb}$ & $3.9(1.8)$ & 2.7 & $\rightarrow \mathrm{H}$ & $\rightarrow \mathrm{H}$ & 0.40 & $2.2(2.2)$ & $2.86(5)$ \\
$\mathrm{Mo}$ & $4.6(0.4)$ & 3.0 & $\rightarrow \mathrm{H}$ & $\rightarrow \mathrm{H}$ & 0.32 & $2.2(2.2)$ & $4(6)$ \\
$\mathrm{Pd}$ & 1.1 & $1.7(1.7)$ & 1.5 & 1.5 & 0 & $2.1(2.1)$ & $0(0)$ \\
$\mathrm{Ag}$ & 0.1 & $0.3(0.2)$ & $\rightarrow \mathrm{A}$ & $\rightarrow \mathrm{A}$ & 0.03 & $2.7(2.5)$ & $0.6(1)$ \\
$\mathrm{Ta}$ & $2.8(2.4)$ & 2.4 & 2.5 & $\rightarrow \mathrm{H}$ & 0.73 & $2.1(2.2)$ & $3.01(3)$ \\
$\mathrm{W}$ & $3.4(0.9)$ & 2.5 & 2.6 & 3.3 & 0.59 & $2.1(2.1)$ & $2.01(6)$ \\
$\mathrm{Pt}$ & $\rightarrow \mathrm{Z}$ & $2.7(2.4)$ & 2.4 & $\rightarrow \mathrm{A}$ & 0 & $2.1(2.1)$ & $0(2)$ \\
$\mathrm{Au}$ & 0.3 & $0.6(0.5)$ & 0.4 & 0.6 & 0.04 & $2.2(2.2)$ & $1.02(1)$ \\
$\mathrm{Al}$ & 1.6 & 1.4 & 1.5 & $\rightarrow \mathrm{H}$ & & 2.3 & \\
$\mathrm{C}$ & $\rightarrow \mathrm{Z}$ & 3.7 & 4.2 & $\rightarrow \mathrm{A}$ & & 1.5 & \\
$\mathrm{Si}$ & 2.5 & 2.2 & 2.5 & 2.2 & & 2.1 & \\
$\mathrm{~Pb}$ & $1.3(0.8)$ & 1.0 & 1.2 & $\rightarrow \mathrm{H}$ & 0.01 & $2.6(2.6)$ & $0(2)$ \\
$\mathrm{H}$ & & & & 2.8 & & 1.1 & \\
$\mathrm{O}$ & & & 5.1 & - & & 1.5 & \\
$\mathrm{~S}$ & $\rightarrow \mathrm{A}$ & 2.8 & 2.4 & $\rightarrow \mathrm{Z}$ & & 1.9 &
\end{tabular}

${ }^{a}$ Binding energies, $E_{\mathrm{b}}^{\mathrm{u}}$, listed in the second through fifth columns are obtained from spin-unpolarized total energies calculated for the fully relaxed atomic structure. The binding energy $E_{\mathrm{b}}^{\mathrm{p}}$ and $\bar{d}_{\mathrm{C}-\mathrm{A}}$ values given in parentheses are obtained from spin-polarized total energies calculated for the fully relaxed structure at the adsorption site where $E_{\mathrm{b}}^{\mathrm{u}}$ has a minimum value. $\Delta E_{\mathrm{T}}$ is the difference between spinunpolarized and spin-polarized total energies as in eq 2. $\mu$ is the calculated magnetic moment ( $\mu_{\mathrm{B}}$ per supercell) of the magnetic ground state of the $(\mathrm{A}+\mathrm{SWNT})$ system. The calculated magnetic moments of free atoms are presented in parentheses. Results for hydrogen and oxygen atoms are taken from refs 20 and $21 . \rightarrow \mathrm{H}$ implies that the adatom at the given site is not stable, and eventually it moves to the $\mathrm{H}$ site.

TABLE 2: Calculated Binding Energies and Average Carbon-Adatom Bond Distances, $d_{\mathrm{C}-\mathrm{A}}$, of Individual Atoms (Ti, Mn, Mo, Au) Adsorbed at H, Z, A, and T Sites of the $(6,6)$ SWNT as Described in Figure $1^{a}$

\begin{tabular}{llllllll}
\hline atom & $\mathrm{H}(\mathrm{eV})$ & $\mathrm{A}(\mathrm{eV})$ & $\mathrm{Z}(\mathrm{eV})$ & $\mathrm{T}(\mathrm{eV})$ & $\Delta E_{\mathrm{T}}(\mathrm{eV})$ & $\bar{d}_{\mathrm{C}-\mathrm{A}}(\AA)$ & $\mu\left(\mu_{\mathrm{B}}\right)$ \\
\hline $\mathrm{Ti}$ & $2.6(1.8)$ & 1.7 & 1.8 & 1.7 & 0.5 & $2.2(2.2)$ & $1.68(4)$ \\
$\mathrm{Mn}$ & $3.3(0.1)$ & $\rightarrow \mathrm{H}$ & $\rightarrow \mathrm{H}$ & $\rightarrow \mathrm{H}$ & 2.2 & $2.1(2.5)$ & $5.60(5)$ \\
$\mathrm{Mo}$ & $4.3(0.1)$ & $\rightarrow \mathrm{H}$ & $\rightarrow \mathrm{H}$ & $\rightarrow \mathrm{H}$ & 0.2 & $2.2(2.3)$ & $3.61(6)$ \\
$\mathrm{Au}$ & 0.2 & 0.3 & $\rightarrow \mathrm{T}$ & $0.4(0.3)$ & 0.02 & $2.3(2.3)$ & $0.79(1)$
\end{tabular}

${ }^{a}$ Binding energies, $E_{\mathrm{b}}^{\mathrm{u}}$, listed in the second through fifth columns are obtained from spin-unpolarized total energies calculated for the fully relaxed atomic structure. The binding energy $E_{\mathrm{b}}^{\mathrm{p}}$ and $\bar{d}_{\mathrm{C}-\mathrm{A}}$ values given in parentheses are obtained from spin-polarized total energies calculated for the fully relaxed structure at the adsorption site where $E_{\mathrm{b}}^{\mathrm{u}}$ has a minimum value. $\Delta E_{\mathrm{T}}$ is the difference between spinunpolarized and spin-polarized total energies as in eq $2 . \mu$ is the calculated magnetic moment $\left(\mu_{\mathrm{B}}\right.$ per supercell) of the magnetic ground state of the $(\mathrm{A}+\mathrm{SWNT})$ system. The calculated magnetic moments of free atoms are presented in parentheses.

to a nonmagnetic state ranges from $\sim 1$ to $\sim 4.5 \mathrm{eV}$. Whereas alkali and simple metals have binding energies in the range of $1.5 \mathrm{eV}$, the chemisorption energy of transition metals is relatively higher. However, metals such as $\mathrm{Cu}, \mathrm{Au}, \mathrm{Ag}$, and $\mathrm{Zn}$ have relatively weak binding. The attractive van der Waals interaction may be important for their stabilization. Group IV elements such as $\mathrm{C}$ and $\mathrm{Si}$ can also be bound with a significant binding energy. The center of the hexagons made by $\mathrm{C}-\mathrm{C}-\mathrm{C}$ bonds on the SWNT surface appears to be favored by most of the adatoms. The average $\mathrm{C}-$ adatom bond distance occurs in the range of $2.0-2.3 \AA$. However, $\bar{d}_{\mathrm{C}-\mathrm{A}}$ is relatively smaller for $\mathrm{H}, \mathrm{C}$, and $\mathrm{O}$ atoms having small atomic radii. It is well known that the interaction between the graphite surface and most of the atoms included in Tables 1 and 2 is actually weak. The curvature effect is the primary factor that strengthens the binding. ${ }^{24,25}$

We note that specific adsorbate-SWNT $(A+$ SWNT) systems are found to be in a magnetic ground state, hence $E_{\mathrm{T}}^{\mathrm{p}}[\mathrm{A}+\mathrm{SWNT}]<E_{\mathrm{T}}^{\mathrm{u}}[\mathrm{A}+\mathrm{SWNT}]$. No matter what the value of the binding energy, a stable binding of a particular A + SWNT geometry is meaningful if it belongs to a ground state. In Tables 1 and 2, we present the differences between the spinunpolarized and spin-polarized total energies:

$$
\Delta E_{\mathrm{T}}=E_{\mathrm{T}}^{\mathrm{u}}[\mathrm{A}+\mathrm{SWNT}]-E_{\mathrm{T}}^{\mathrm{p}}[\mathrm{A}+\mathrm{SWNT}]
$$

Here, $\Delta E_{\mathrm{T}}>0$ indicates that the magnetic ground state with a net spin is favored. These Tables are crucial for the further study of SWNT coverage with foreign atoms (such as Sc, Co, Ti, $\mathrm{Nb}, \mathrm{Ta}$ ) to generate magnetic nanostructure.

We can extract the following useful information from the results of calculations listed in these Tables. In general, the binding energies calculated for the nonmagnetic state are higher than those corresponding to the magnetic ground state. This is partially due to the reference energies in eq 1 . Most of the transition-metal atoms adsorbed on the $(8,0)$ and $(6,6)$ SWNTs have a magnetic ground state with $\Delta E_{\mathrm{T}}>0$, and hence they give rise to a net magnetic moment ranging from $5.49 \mu_{\mathrm{B}}$ (for $\mathrm{Mn}$ ) to zero magnetic moment (for $\mathrm{Pd}$ and $\mathrm{Pt}$ ). Although the Ni-adsorbed SWNT has a very low magnetic moment $\left(0.04 \mu_{\mathrm{B}}\right)$, adsorbates such as $\mathrm{Au}, \mathrm{Ag}$, or $\mathrm{Cu}$ have magnetic moments in the range of $0.4-0.6 \mu_{\mathrm{B}}$. Our spin-polarized and spin-unpolarized calculations show that these transition-metal atoms in Tables 1 and 2 also have a magnetic ground state when they are free. Because a bare SWNT has a nonmagnetic ground state, the net spin of the A + SWNT system originates from the magnetic moment of the adsorbed atom. The calculated magnetic moments of these free atoms are in good agreement with the values given by Moore. ${ }^{30}$ However, atoms such as $\mathrm{Na}, \mathrm{Al}, \mathrm{C}$, $\mathrm{Si}, \mathrm{Pb}, \mathrm{O}, \mathrm{S}$, and $\mathrm{H}$ favor the nonmagnetic ground state when adsorbed on the $(8,0)$ SWNT. The magnetic moment generated upon the adsorption of individual transition atoms has important implications and points to the issue of whether molecular magnets (or nanomagnets) can be produced from carbon nanotubes. Addressing this issue may open an active field of study on SWNTs, which are covered or substitutionally doped by transition-metal atoms according to a well-defined pattern. The implementation of transition-metal elements inside the tube is another way to obtain nanomagnetic structures. In this way, these atoms are prevented from oxidation. Whether a permanent magnetic moment by the exchange interaction can be generated on these transition-metal-coated SWNTs would be an interesting question to answer. Recently, the magnetization and hysteresis loops of iron nanoparticles partially encapsulated at the tips and inside of aligned carbon nanotubes have been demonstrated by experiment. ${ }^{19}$

Figure 2 presents the variation of binding energies $E_{\mathrm{b}}^{\mathrm{u}}$ and $E_{\mathrm{b}}^{\mathrm{p}}$ of the first row transition-metal elements with respect to the number of d electrons $N_{\mathrm{d}}$. Earlier, we showed that the variation of $E_{\mathrm{b}}^{\mathrm{p}}\left(N_{\mathrm{d}}\right)$ with $N_{\mathrm{d}}$ display similar behavior shown by the bulk 


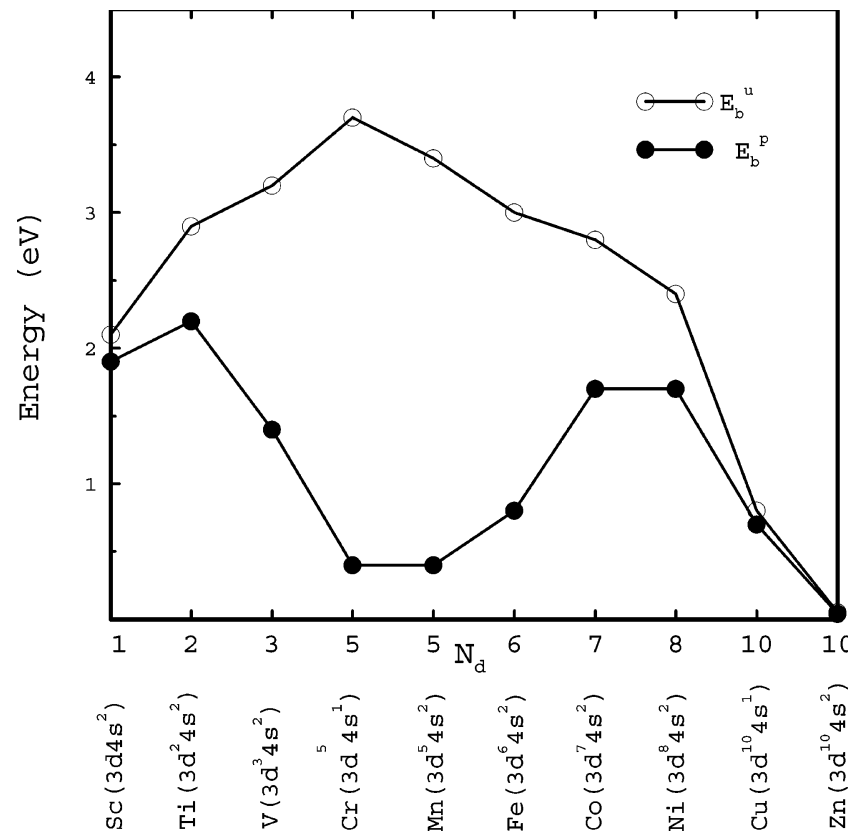

Figure 2. Variation of the calculated spin-unpolarized $E_{\mathrm{b}}^{\mathrm{u}}$ and spinpolarized $E_{\mathrm{b}}^{\mathrm{p}}$ binding energies of transition-metal atoms (adsorbed on the $(8,0)$ tube) with respect to the number of d electrons, $N_{\mathrm{d}}$.

cohesive energies $E_{\mathrm{c}}\left(N_{\mathrm{d}}\right)$ and bulk modulus $B\left(N_{\mathrm{d}}\right) \cdot{ }^{22} E_{\mathrm{c}}\left(N_{\mathrm{d}}\right)$ and $B\left(N_{\mathrm{d}}\right)$ having a minimum at $N_{\mathrm{d}}=5$ between two maximum value were explained by the Friedel model. ${ }^{31}$ We see that whereas the $E_{\mathrm{b}}^{\mathrm{p}}\left(N_{\mathrm{d}}\right)$ curve corresponding to the magnetic ground state follows this trend the variation of $E_{\mathrm{b}}^{\mathrm{u}}\left(N_{\mathrm{d}}\right)$ is dramatically different.

Binding energies listed in Tables 1 and 2 are of particular interest for the coating of SWNTs by metal atoms and hence for the fabrication of nanowires. The atoms that were observed to form continuous and quasicontinuous coatings on the SWNT ( $\mathrm{Ti}, \mathrm{Ni}, \mathrm{Pd}$ ) have relatively higher binding energies as compared to those atoms $(\mathrm{Au}, \mathrm{Fe}, \mathrm{Pb})$ that form only discrete particles on the surface of the tube. ${ }^{11} \mathrm{We}$ also note that in forming a good coverage not only adatom-SWNT interaction but also other factors, possibly adatom-adatom interaction, play a crucial role. ${ }^{17}$ Good conductors such as $\mathrm{Au}, \mathrm{Ag}$, and $\mathrm{Cu}$ have very weak binding. However, $\mathrm{Na}$ with one $3 \mathrm{~s}$ electron on the outer shell is bound with a significant binding energy $\left(E_{\mathrm{b}}=1.3 \mathrm{eV}\right)$. The binding energy of $\mathrm{Mg}$ is very weak and is only $0.03 \mathrm{eV}$ at the $\mathrm{H}$ site because of its outer shell $(3 \mathrm{~s})^{2}$. For the same reason, $\mathrm{Zn}$ by itself exhibits a similar trend $\left(E_{\mathrm{b}}^{\mathrm{u}}=0.05 \mathrm{eV}\right)$ with its $(4 \mathrm{~s})^{2}$ valence structure. Owing to the weak binding, the type of bond between $\mathrm{Mg}(\mathrm{Zn})$ and the SWNT is between chemisorption and physisorption with $d_{\mathrm{C}-\mathrm{A}}=3.8 \AA\left(d_{\mathrm{C}-\mathrm{A}}=3.7 \AA\right)$. Although an individual $\mathrm{Al}$ atom (with a $(3 \mathrm{~s})^{2} 3 \mathrm{p}$ valence structure) is not bound to the graphite surface, its binding on the $(8,0)$ SWNT is relatively strong. This can be explained by the curvature effect because the binding was found to be even stronger at the highcurvature site of the SWNT under uniaxial radial deformation. ${ }^{24,25}$ Here we point out an interesting trend between Tables 1 and 2. The binding energies $E_{\mathrm{b}}^{\mathrm{u}}$ as well as $E_{\mathrm{b}}^{\mathrm{p}}$ and the magnetic moments of the adatoms adsorbed on the $(8,0)$ SWNT came out to be consistently higher than those of the adatom adsorbed on the $(6,6)$ tube. Perhaps this trend can also be explained by the curvature effect. ${ }^{24,32}$

The transition-metal atoms with few d electrons, such as Sc, $\mathrm{Co}, \mathrm{Ti}, \mathrm{Nb}$, and $\mathrm{Ta}$, form strong bonds with binding energies ranging from 2.4 to $1.8 \mathrm{eV}$ and hence can be suitable for the metal coating of SWNTs. These metals can also be used as a buffer layer to form a uniform coating of good conductors such as $\mathrm{Au}, \mathrm{Ag}$, and $\mathrm{Cu}$. Most of the adatoms that we studied yield the strongest binding at the $\mathrm{H}$ site. $\mathrm{Ni}, \mathrm{Pd}$, and $\mathrm{Pt}$ (column VIII elements) and $\mathrm{Cu}, \mathrm{Ag}$, and $\mathrm{Au}$ (column IB elements) seem to prefer the A site. The average adsorbate $-\mathrm{C}$ distance, $\bar{d}_{\mathrm{C}-\mathrm{A}}$, ranges from 1.9 (minimum) to $3.8 \AA$ (maximum); most of them occur at $\sim 2.1 \AA$.

\section{Interaction of Group IV Elements with SWNTs}

The interaction of group IV elements with SWNTs is crucial because they have the same valence configuration as the carbon atoms in nanotubes. Here we consider the parent $\mathrm{C}$ atom and $\mathrm{Si}$ atom. These atoms form rather strong bonds with the SWNT. The calculated binding energies are rather high (i.e., $E_{\mathrm{b}}=4.2$ and $2.5 \mathrm{eV}$, respectively). The $\mathrm{Z}$ site is energetically favorable for both $\mathrm{C}$ and $\mathrm{Si}$. The character of the bond will be clarified in section $\mathrm{V}$.

The substitution of $\mathrm{Si}$ is of particular interest because $\mathrm{SiC}$ is a stable crystal. The $\mathrm{Si}$ substitution can be realized by replacing one of the carbon atoms of the $(8,0)$ SWNT with Si and subsequently by relaxing carbon atoms and $\mathrm{Si}$ until practically zero force on all of these atoms is achieved. To get an idea of the energetics of Si substitution, we calculated the self-consistent total energies of two different systems. The first system, consisting of a (structure-optimized) bare $(8,0)$ SWNT and a single $\mathrm{Si}$ atom placed at the farthest point from the SWNT in the supercell, has a total energy of $E_{\mathrm{T}}[\mathrm{SWNT}, \mathrm{Si}]$. The second system, corresponding to a structure-optimized, Si-substituted SWNT and a single $\mathrm{C}$ atom placed at the farthest point, has a total energy of $E_{\mathrm{T}}[\mathrm{SWNT}(\mathrm{Si}), \mathrm{C}]$. The structure-optimized Sisubstituted SWNT [SWNT(Si)] has been found to be stable. The difference in energy, $\Delta E=E_{\mathrm{T}}[\mathrm{SWNT}(\mathrm{Si}), \mathrm{C}]-E_{\mathrm{T}}[\mathrm{SWNT}$, $\mathrm{Si}$, can give an idea of the energy involved in Si substitution. We found that $\Delta E$ is positive $(\sim 6 \mathrm{eV})$, and hence the substitution of $\mathrm{Si}$ is energetically unfavorable and corresponds to a local minimum in the Born-Oppenheimer surface. Following the definition by Baierle et al., ${ }^{33}$ the substitution energy of a single $\mathrm{Si}$ (i.e., $E_{\mathrm{S}}=E_{\mathrm{T}}[\mathrm{SWNT}(\mathrm{Si})]-E_{\mathrm{T}}[\mathrm{SWNT}]-\left(\mu_{\mathrm{Si}}\right.$ $\left.+\mu_{\mathrm{C}}\right)$ ) is calculated to be $E_{\mathrm{S}}=2.75 \mathrm{eV}$ by using the bulk cohesive energies of $\mathrm{Si}$ and $\mathrm{C}$ for $\mu_{\mathrm{Si}}$ and $\mu_{\mathrm{C}}$, respectively. This energy is comparable to the substitution energy of Si calculated for the $(10,0)$ and $(6,6)$ SWNTs. ${ }^{33}$ If we use the adsorption energies of $\mathrm{Si}$ and $\mathrm{C}$ in Table 1 for $\mu_{\mathrm{Si}}$ and $\mu_{\mathrm{C}}$, then the formation energy is found to be $4.67 \mathrm{eV}$. This latter energy $\left(E_{\mathrm{S}}=4.67\right.$ $\mathrm{eV}$ ) is closer to $\Delta E=6 \mathrm{eV}$ than the previous energy $\left(E_{\mathrm{S}}=\right.$ $2.75 \mathrm{eV}$ ). Nevertheless, both substitution energies calculated by using different definitions indicate that the substitution of $\mathrm{C}$ in SWNT by $\mathrm{Si}$ is unfavorable energetically, hence it is endothermic.

The strong bond formation between $\mathrm{C}$ and SWNTs suggests that two SWNTs can be connected by $\mathrm{C}$ adatoms. We examined the system of two parallel $(8,0)$ SWNTs with a $\mathrm{C}$ atom placed at the mutual A sites of these tubes. A system consisting of two parallel $(8,0)$ SWNTs connected by a $\mathrm{C}$ adatom in a supercell has been relaxed. The binding energy of the additional $\mathrm{C}$ atom connecting the tubes has been calculated from the following relation

$$
\begin{array}{r}
E_{\mathrm{b}}=E_{\mathrm{T}}[\mathrm{C}]+E_{\mathrm{T}}[\mathrm{SWNT}, \mathrm{SWNT}]- \\
E_{\mathrm{T}}[\mathrm{SWNT}+\mathrm{C}+\mathrm{SWNT}]
\end{array}
$$

and has been found to be $4.6 \mathrm{eV}$. The atomic configuration and the local density of states (LDOS) of two fully relaxed SWNTs connected by a $\mathrm{C}$ atom are shown in Figure 3. 


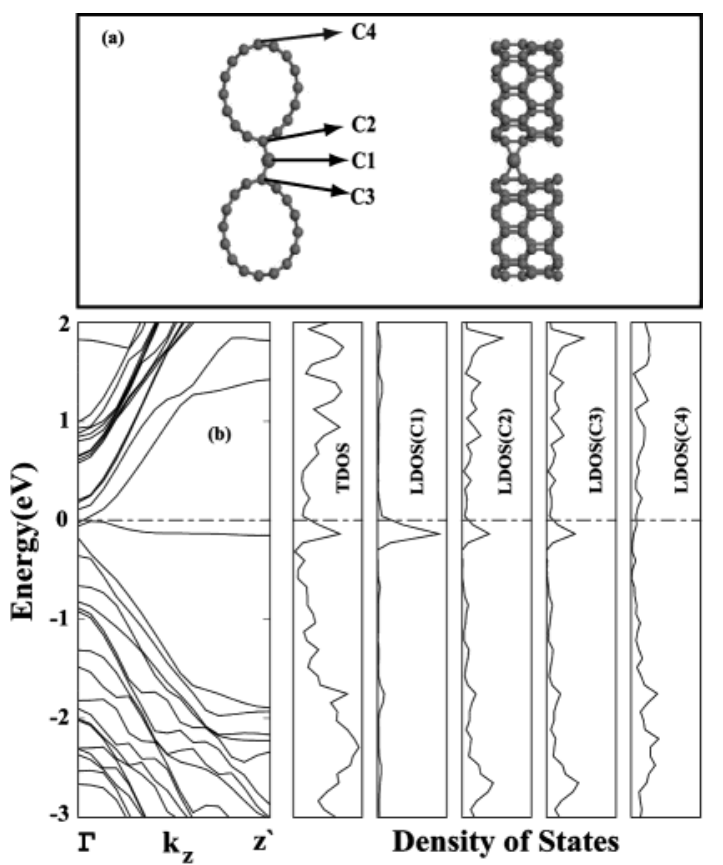

Figure 3. (a) Side views of atomic configuration of two $(8,0)$ SWNTs connected by a carbon adatom (per supercell) located at the mutual A sites. C1, C2, C3, and C4 indicate specific atoms where LDOSs are calculated. These are the connecting carbon adatom $(\mathrm{C} 1)$, the $\mathrm{C}$ atoms $(\mathrm{C} 2, \mathrm{C} 3)$ of the SWNT that form bonds with $\mathrm{C} 1$, and the $\mathrm{C}$ atom of the SWNT that is farthest from the region where two SWNTs are connected (C4). (b) Energy-band structure. TDOS and LDOSs at C1, $\mathrm{C} 2, \mathrm{C} 3$, and C4. Zero of energy is taken at the Fermi level.

The carbon atom between the tubes form four directional bonds - two bonds to the $\mathrm{C}$ atoms of the $\mathrm{C}-\mathrm{C}$ axial bonds of each SWNT. We note a slight deformation and deviation from the circular cross section of the SWNT, where the C adatom is attached. LDOS calculated at the connecting carbon adatom and at the carbon atoms of SWNTs shows a finite density of states at the Fermi level. A flat band derived from the $\mathrm{C}-\mathrm{C}$ bonds overlaps with the lowest conduction band and hence metallizes the whole system as described in Figure 3.

\section{Character of the Bond between the Adsorbed Atom and the SWNT}

In this section, the character of the bond between the adatom and the SWNT has been examined by charge-density analysis. For the fully relaxed, minimum total-energy configuration, we calculated the total and difference charge densities. The total charge density is

$$
\rho(\boldsymbol{r})=\sum_{n, k}^{\text {occup }} \Psi_{n, k}^{*}(\boldsymbol{r}) \Psi_{n, k}(\boldsymbol{r})
$$

and the difference charge density is

$$
\Delta \rho(\boldsymbol{r})=\rho[\mathrm{A}+\mathrm{SWNT}]-\rho[\mathrm{SWNT}]-\rho[\mathrm{A}]
$$

In the latter equation, the total charge densities of adatomadsorbed SWNTs, $\rho[\mathrm{A}+\mathrm{SWNT}]$, of bare SWNTs, $\rho$ [SWNT], and of the free atom, $\rho[\mathrm{A}]$, all are calculated in the same supercell with the same atomic positions described in eq 1 . Thereafter, the difference charge density conveys information about the charge rearrangements upon adsorption. In Figure 4, we show $\rho(\boldsymbol{r})$ and $\Delta \rho(\boldsymbol{r})$ calculated for four adatoms, namely, $\mathrm{Na}, \mathrm{Al}, \mathrm{C}$, and Ti. We did not present the counterplots of $\rho(\boldsymbol{r})$ for $[\mathrm{Na}+\mathrm{SWNT}]$ because they do not convey relevant
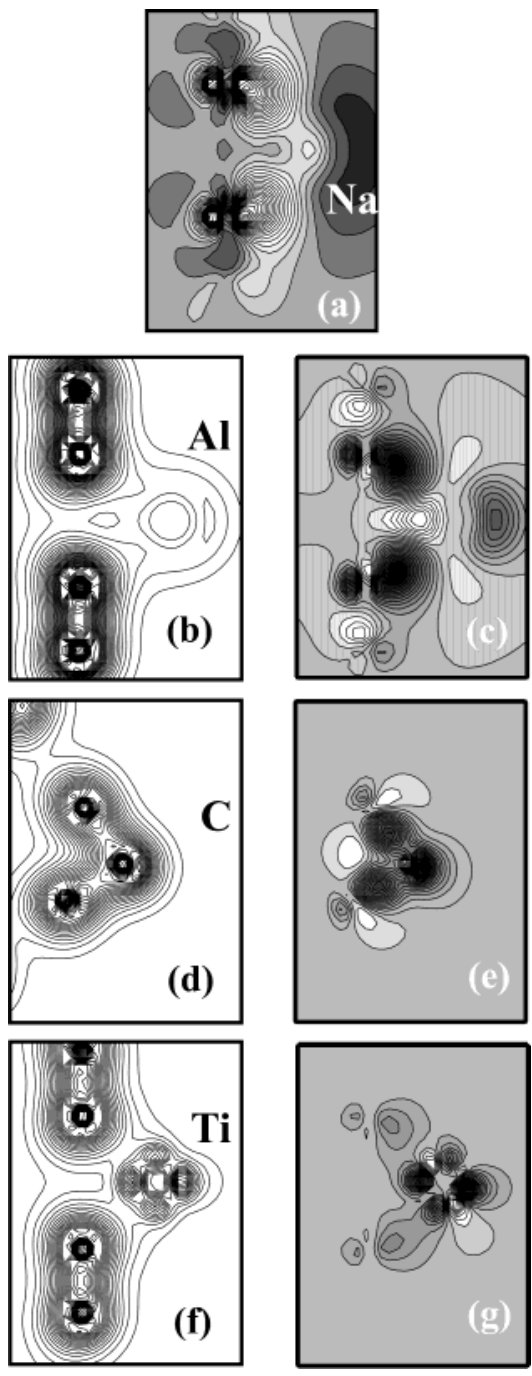

Figure 4. Contour plots of total $\rho(\boldsymbol{r})$ and difference $\Delta \rho(\boldsymbol{r})$ charge densities. For $\mathrm{C}$, the charge densities are calculated on a plane passing through adatom and zigzag $\mathrm{C}-\mathrm{C}$ bond. For others $(\mathrm{Na}, \mathrm{Al}, \mathrm{Ti})$, the charge-density plane passes through the center of the hexagon and adatom. In contour plots of $\Delta \rho(\boldsymbol{r})$, charge is depleted from black regions and is accumulated at white regions.

information owing to the low charge density around the adsorbed $\mathrm{Na}$ atom. Dramatic differences in the valence electron configuration of these adatoms are reflected in the character of the bonds they form with SWNTs.

Our analysis shows that charge is generally transferred from the adatom and the $\mathrm{C}-\mathrm{C}$ bond of an SWNT. The $3 \mathrm{~s}$ valence state of $\mathrm{Na}$ is weakly bound and is donated to the conduction band of the SWNT. Therefore, the band is modified. In the case of $\mathrm{Al}$, which is adsorbed at the $\mathrm{H}$ site, electrons are transferred from $\mathrm{Al}$ and the nearest $\mathrm{C}$ atoms to the region between the $\mathrm{Al}$ atom and the center of the hexagon on the surface of the SWNT and to the $\mathrm{C}-\mathrm{C}$ bond. The character of the bond formed between the adatom carbon and the $\mathrm{C}$ atoms of the SWNT is reminiscent of the $\mathrm{sp}^{3}$ covalent bonds in diamond crystals. $\rho(\boldsymbol{r})$ has a maximum value at the center of the $\mathrm{C}-\mathrm{C}$ bonds. Because of new bond formation, charge is transferred to the back bonds. The charge density of adsorbed Si (not shown in Figure 4) exhibits character similar to that of adsorbed C. Directional bonds form between $\mathrm{Si}$ and $\mathrm{C}$ atoms. The Ti atom adsorbed at the $\mathrm{H}$ site forms a bond. The $\mathrm{d}$ orbitals are responsible for this bond. According to the Mulliken analysis, the transfer of charge from the Ti atom is 1.45 electrons for the spin-unpolarized case. 

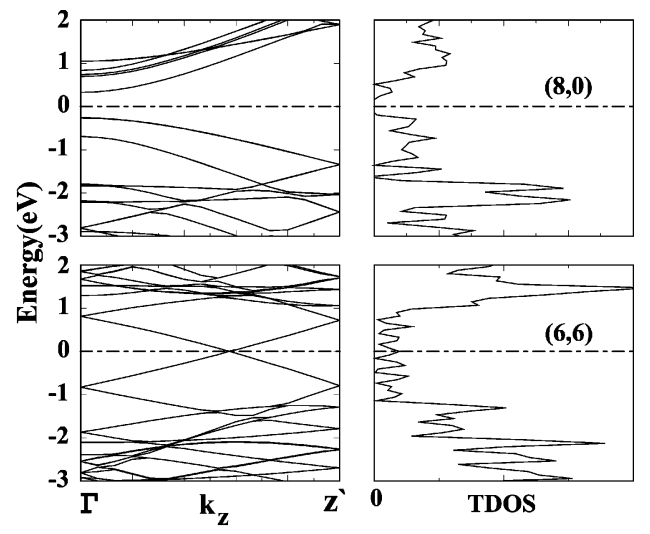

Figure 5. Energy-band structures and total density of states (TDOS) of bare tubes with fully relaxed atomic structure. Electronic structure of the semiconducting $(8,0)$ zigzag SWNT calculated for the double primitive unit cells consisting of $64 \mathrm{C}$ atoms. Electronic structure of the metallic $(6,6)$ armchair SWNT calculated for the quadruple primitive unit cells including $96 \mathrm{C}$ atoms. Zero of energy is set at the Fermi level, $E_{\mathrm{F}}$.

The interaction between the Au atom and the SWNT is weak, which results in a small binding energy. Accordingly, the charge rearrangement as a result of $\mathrm{Au}$ adsorption is minute.

\section{Electronic Structure of the Adatom-SWNT System}

The modification of electronic structure induced by the adsorbed foreign atoms is of prime interest in this study. We analyze the induced modifications by energy-band structure and the local and total density of states obtained for fully relaxed structures. An individual atom adsorbed on an SWNT may give rise to resonance states in the valence and conduction bands and also localized states in the band gaps. Owing to the supercell method used in this study, the energy states associated with a single adsorbate form energy bands, however. Actually, these bands correspond to a linear chain of adsorbates with a 1D lattice constant, $c_{\mathrm{s}}$. The dispersion or width of the bands is a measure of adsorbate-adsorbate coupling. To reduce the adsorbateadsorbate coupling, which is a spurious effect of the supercell method, we have considered one adsorbate per two unit cells for the $(8,0)$ zigzag SWNT (i.e., $c_{\mathrm{s}} \approx 2 c$, which corresponds to a nearest-neighbor distance of $8.52 \AA$ between two adsorbates) and one adsorbate per four unit cells for the $(6,6)$ armchair SWNT (i.e., $c_{\mathrm{s}} \approx 4 c$, which corresponds to a nearest-neighbor distance of $9.86 \AA$ between two adsorbates). To deduce the changes in the electronic structure of the bare tubes induced by the adsorbates, we first present in Figure 5 the electronic structure and total density of states (TDOS) of the bare $(8,0)$ and bare $(6,6)$ SWNTs calculated for double and quadruple primitive unit cells, respectively. Note that upon enlarging the unit cells for 2 and 4 times, the corresponding Brillouin zone is reduced by $1 / 2$ and $1 / 4$, respectively. Accordingly, the energy bands are zone-folded. We see that the direct band gap of the $(8,0)$ zigzag SWNT occurs at the center of the Brillouin zone, but the $(6,6)$ armchair tube is a metal with bands crossing at $E_{\mathrm{F}}$.

A. Semiconducting $(8,0)$ SWNT. The localized states are relevant to the doping of a semiconducting SWNT. Depending on their position relative to the band edges, they are specified as donor states (if they are close to the edge of the conduction band, $E_{\mathrm{C}}$ ) or as acceptor states (if they can occur close to the edge of the valence band, $E_{\mathrm{V}}$ ). The latter case is also known as hole doping. Whether the associated localized state is a donor or acceptor state can be inferred from the energy position of

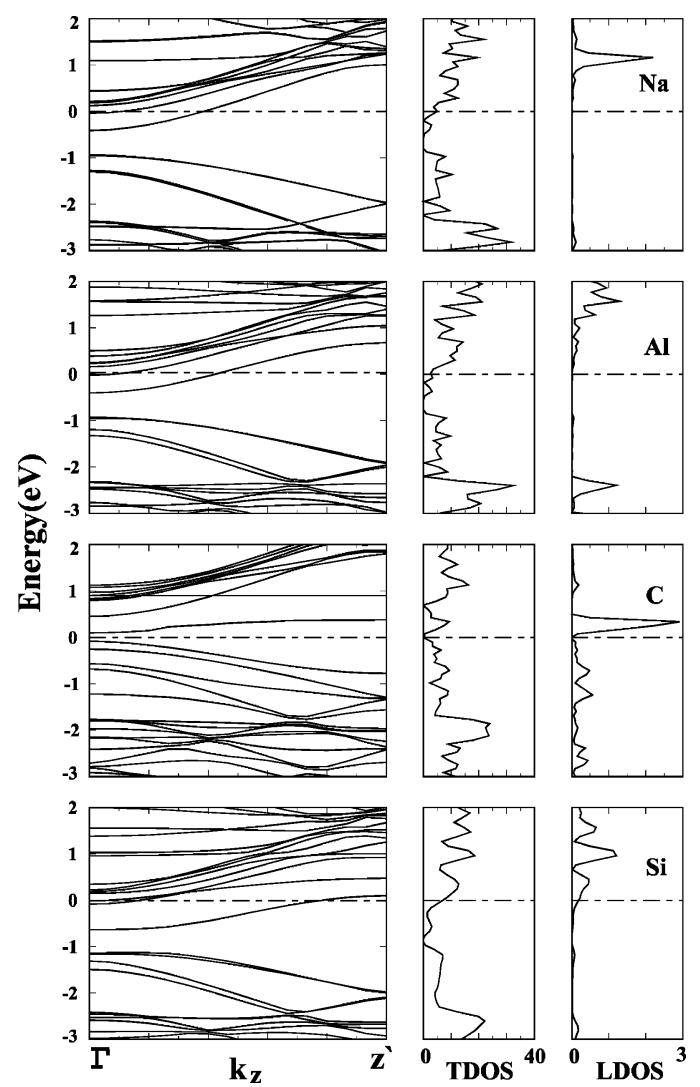

Figure 6. Energy-band structures and TDOSs and LDOSs of single $\mathrm{Na}, \mathrm{Al}, \mathrm{C}$, and $\mathrm{Si}$ adsorbed on a zigzag $(8,0)$ tube. LDOSs were calculated at the adsorbate. Zero of energy is set at the Fermi level. $\mathrm{Na}, \mathrm{Al}$, and $\mathrm{Si}$ are adsorbed at the $\mathrm{H}$ site; $\mathrm{C}$ is adsorbed at the $\mathrm{Z}$ site.

the band in the gap. We first consider $\mathrm{Na}, \mathrm{Al}, \mathrm{C}$, and $\mathrm{Si}$, which are adsorbed individually on the $(8,0)$ SWNT with a repeat period of $2 c$ and give rise to the spin-paired, nonmagnetic ground state. In Figure 6, we present the calculated band structures and LDOS at the adatoms.

The calculated band structure of an SWNT with Na adsorbed at the $\mathrm{H}$ site forming a regular chain with a lattice parameter (or interatomic distance) of $a=c_{\mathrm{s}} \approx 2 c$ yields a half-filled band. This band is normally the first empty conduction band of the tube. Because the $4 \mathrm{~s}$ valence electron of $\mathrm{Na}$ has low ionization energy, it is donated to the SWNT to occupy the empty conduction band. As a result, this empty band is gradually populated and also modified upon the adsorption of $\mathrm{Na}$, hence the SWNT becomes metallized. Similarly, the calculated band structure of the SWNT with $\mathrm{Al}$ adsorbed at the $\mathrm{H}$ site forming a regular chain structure gives rise to a half-filled band derived from the empty conduction band of the SWNT. Therefore, the localized state due to an adsorbed individual $\mathrm{Al}$ atom is a donor state.

In the case of $\mathrm{C}$, a small gap occurs between the bands derived from adsorbate states. The empty and filled bands just above and below $E_{\mathrm{F}}$ are derived from the $\mathrm{C}$ adatom with a significant contribution from the SWNT. Si yields an almost fully occupied band in the band gap and an almost empty band at the bottom of the conduction band. The dispersion of bands for Si adsorbed on the $\mathrm{H}$ site displays differences from that of the $\mathrm{C}$ adatom adsorbed on the $\mathrm{Z}$ site. However, the overall behavior of the state densities is similar.

In Figure 7, the spin-polarized band structure and TDOS of adsorbed transition-metal atoms (i.e., Au, Mn, Mo, Ti) display a different situation because of the magnetic ground state. Au yields two bands in the band gap of bare SWNT; these are filled 


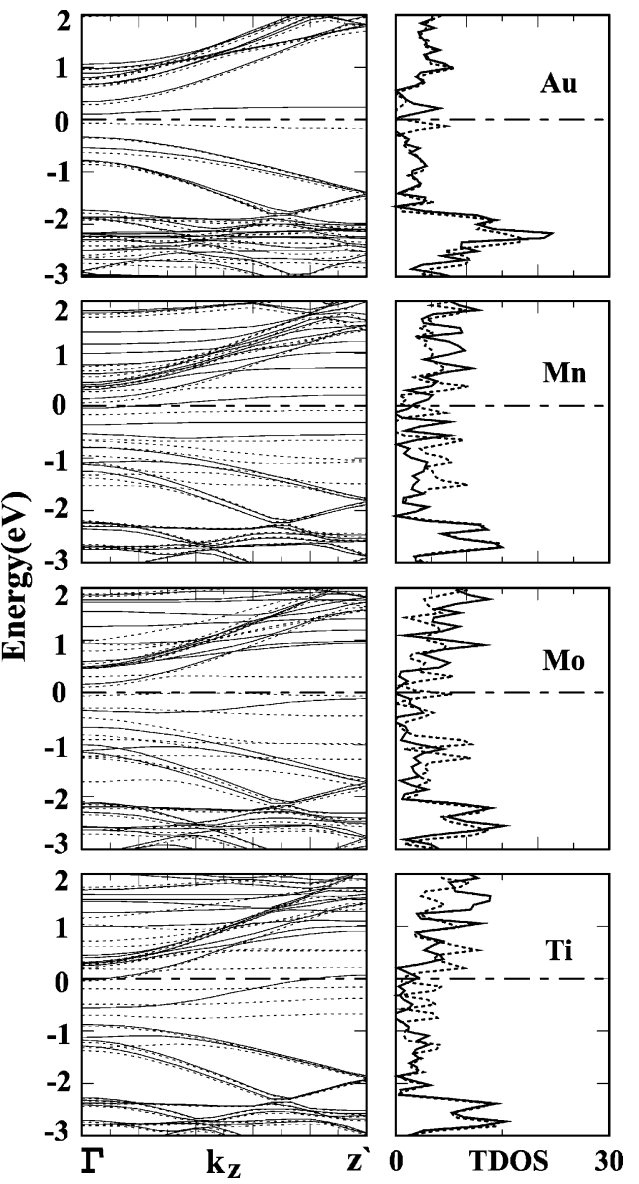

Figure 7. Energy-band structures and total density of states (TDOS) of single $\mathrm{Au}, \mathrm{Mn}, \mathrm{Mo}$, and $\mathrm{Ti}$ adsorbed on a zigzag $(8,0)$ tube. Zero of energy is set at the Fermi level. Bands and state density of spin-up and spin-down states are shown by dotted and continuous lines, respectively. $\mathrm{Mn}, \mathrm{Mo}$, and $\mathrm{Ti}$ are adsorbed at the $\mathrm{H}$ site; $\mathrm{Au}$ is adsorbed at the $\mathrm{T}$ site.

Au spin-up and empty spin-down bands. This is in compliance with the calculated magnetic moment of $1.02 \mu_{\mathrm{B}}$ per adsorbed $\mathrm{Au}$ atom. There is a small band gap of $\sim 0.2 \mathrm{eV}$ between these Au bands. Comparing these bands with those of the bare $(8,0)$ SWNT in Figure 5, we see that the adsorption of Au did not induce a significant modification in the bands of $(8,0)$, except that the Au $6 s(\uparrow)$ and $\mathrm{Au} 6 \mathrm{~s}(\downarrow)$ bands in the gap gave rise to two sharp peaks below and above $E_{\mathrm{F}}$, respectively, in TDOS. That the contribution of SWNT states to these peaks is minute and the band gap between the conduction and valence bands of the SWNT is practically unchanged confirms the weak interaction between $\mathrm{Au}$ and the SWNT. Filled $\mathrm{Au} 5 \mathrm{~d}(\uparrow)$ and $5 \mathrm{~d}(\downarrow)$ bands occur in the valence band of SWNT $2 \mathrm{eV}$ below $E_{\mathrm{F}}$. The free Mn atom normally has a $\left(3 \mathrm{~d}^{5}\right)\left(4 \mathrm{~s}^{2}\right)$ configuration and a magnetic moment of $5 \mu_{\mathrm{B}}$. The flat spin-up and spin-down bands of $\mathrm{Mn}$ occur in the band gap and near the edge of the valence band. The band gap between the highest occupied spin-up and lowest empty spin-down band is very small $(<0.1 \mathrm{eV})$. The $\mathrm{d}$ bands of adsorbed Mo occur in the band gap and near the top of the valence band of the SWNT. The band gap between the highest occupied spin-up band and the lowest spin-down band is $\sim 0.2$ $\mathrm{eV}$. Thus, the highest occupied state due to Mo occurs near the edge of the conduction band. The number of $4 \mathrm{~d}(\uparrow)$ bands confirms that the net magnetic moment of the Mo-adsorbed SWNT is $4 \mu_{\mathrm{B}}$ per cell. Three bands formed from Ti $3 \mathrm{~d}(\uparrow)$ are fully occupied and accommodate three electrons of the adsorbed Ti atom. Other Ti $3 \mathrm{~d}(\uparrow)$ bands occur above $E_{\mathrm{F}}$, but they overlap with the conduction band of the SWNT. The dispersive and

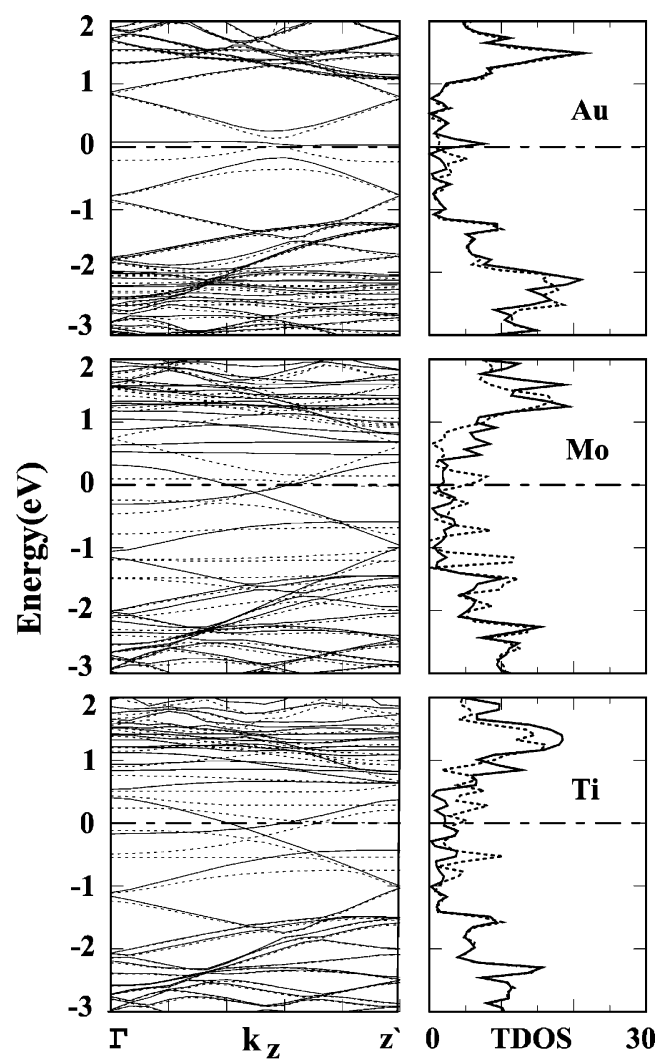

Figure 8. Energy-band structures and total density of states (TDOS) of single $\mathrm{Au}, \mathrm{Mo}$, and $\mathrm{Ti}$ adsorbed on a armchair $(6,6)$ tube. Zero of energy is set at the Fermi level. Bands and state density of spin-up states and spin-down states are shown by dotted and continuous lines, respectively. Mo and $\mathrm{Ti}$ are adsorbed at the $\mathrm{H}$ site; $\mathrm{Au}$ is adsorbed at the $\mathrm{T}$ site.

almost fully occupied spin-down band is formed from the states of carbon and hence is derived from the conduction band of the bare SWNT. The SWNT is metallized upon Ti adsorption because this band crosses the Fermi level and also overlaps with the other conduction bands. This situation is in accordance with the Mulliken analysis, which predicts that electrons are transferred from Ti to the SWNT.

B. Metallic $(6,6)$ SWNT. As far as the adsorption of foreign atoms is concerned, the main difference between the semiconducting $(8,0)$ tube and the metallic $(6,6)$ tube has been the relatively lower binding energies calculated for the adsorption on the latter. This trend has been attributed to the curvature effect. In this section, we explore whether the metallic nature of the tube can influence the electronic structure in any essential manner. In Figure 8, the energy-band structures of Au, Mo, and Ti adsorbed on the $(6,6)$ tube are shown. In the case of $\mathrm{Au}$, the band structure of the bare $(6,6)$ tube is not affected except that a small gap is opened between the $\pi$-valence and $\pi^{*}$-conduction bands crossing at $E_{\mathrm{F}}$. The gap opening is a direct consequence of the symmetry as explained by Delenay et al. ${ }^{34}$ In this gap, we see Au spin-up and Au spin-down bands, which give rise to two peaks on both sides of $E_{\mathrm{F}}$. The overall behavior of TDOS near $E_{\mathrm{F}}$ appears to be similar for the adsorption of $\mathrm{Au}$ on both the $(8,0)$ and $(6,6)$ tubes. We see, however, significant modifications in the band structure of bare $(6,6)$ upon the adsorption of Mo and Ti. Although the overall behavior of state densities near $E_{\mathrm{F}}$ is similar for the adsorption of individual Mo and Ti on both the $(8,0)$ and $(6,6)$ tubes, a very small band gap between the spin-up and spin-down bands of Mo adsorbed in the $(8,0)$ tube disappears when it is adsorbed on the $(6,6)$ tube. 


\section{Discussions and Conclusions}

Our study shows that interesting physical properties can be generated by the adsorption of a single atom on an SWNT. Changes in the physical properties depend on the valency of the adsorbate. Na having a $3 \mathrm{~s}$ valence state with a small ionization potential donates this valence electron to the empty conduction band of a semiconducting SWNT. Therefore, the adsorption of $\mathrm{Na}$ results in the metallization of the $(8,0)$ tube. Similar effects occur upon the adsorption of Al. However, the adsorption of individual transition-metal atoms gives rise to dramatically different results. Because of their occupied d states, the transition-metal-adsorbed SWNT has a magnetic ground state. In most of the cases, an adsorbed individual transitionmetal atom gives rise to a band gap between the spin-up and spin-down bands within the supercell geometry. Ti, which leads to a metal, appears to be an exception. Another important property that is specific to $\mathrm{Ti}$ is its continuous coating of SWNTs. This should be related to both Ti-Ti and Ti-SWNT interactions. Whether the Ti-covered SWNT leads to regular atomic structure and the nanowire produced from a Ti-covered SWNT allows ballistic quantum transport are important issues requiring further investigation. Trends in the adsorption of transition-metal atoms on the $(6,6)$ and $(8,0)$ tubes are similar, except that the binding energies in the former are consistently smaller. We attribute this behavior to the relatively smaller curvature of the $(6,6)$ tube.

Higher coverage and decoration of adsorbed foreign atoms can produce nanostructures (such as nanomagnets, nanometersized magnetic domains, 1D conductors and thin metallic connects, and electronic devices) that may find interesting technological applications, in spintronics, high-density data storage, and interconnects between devices. The d orbitals of the transition-metal atoms are responsible for relatively higher binding energies, which display an interesting variation with the number of filled d states.

Acknowledgment. This work was partially supported by the NSF under grant no. INT01-15021 and TÜBÍTAK under grant no. TBAG-U/13(101T010). We thank Professor S. Suzer for stimulating discussions. S.C. acknowledges partial support from TUBA (Academy of Science of Turkey).

\section{References and Notes}

(1) Iijima, S. Nature (London) 1991, 354, 56. Iijima, S.; Ichihashi, T.; Ando, Y. Nature (London) 1992, 356, 776.

(2) Dresselhaus, M. S.; Dresselhaus, G.; Eklund, P. C. Science of Fullerenes and Carbon Nanotubes; Academic Press: San Diego, CA, 1966.

(3) Agraït, N.; Rodrigo, J. G.; Vieira, S. Phys. Rev. B 1993, 47, 12345.

(4) Ohnishi, H.; Kondo, Y.; Takayanagi, K. Nature (London) 1998, $395,783$.

(5) Yanson, A. I.; Bollinger, G. R.; Brom, H. E.; Agraï, N.; Ruitenbeek, J. M. Nature (London) 1998, 395, 783.
(6) Ciraci, S.; Tekman, E. Phys. Rev B 1989, 40, R11969. Tekman, E.; Ciraci, S. Phys. Rev. B 1991, 43, 7145.

(7) Ciraci, S.; Buldum, A.; Batra, I. P. J. Phys.: Condens. Matter 2001, 13, R537.

(8) Buldum, A.; Ciraci, S.; Fong, C. Y. J. Phys.: Condens. Matter 2000, 12, 3349. Buldum, A.; Leitner, D. M.; Ciraci, S. Europhys. Lett. 1999, $47,208$.

(9) Ozpineci, A.; Ciraci, S. Phys. Rev. B 2001, 63, 125415.

(10) Gülseren, O.; Ercolesi, F.; Tosatti, E. Phys. Rev. Lett. 1998, 80, 3775. Tosatti, E.; Prestipino, S. Science 2000, 289, 561.

(11) Dai, H.; Wong, E. W.; Lu, Y. Z.; Fan, S.; Lieber, C. M. Nature (London) 1995, 375, 769. Han, W. Q.; Fan, S. S.; Li, Q. Q.; Hu, Y. D. Science 1997, 277, 1287.

(12) Zhang, Y.; Dai, H. Appl. Phys. Lett. 2000, 77, 3015. Zhang, Y.; Franklin, N. W.; Chen, R. J.; Dai, H. Chem. Phys. Lett. 2000, 331, 35.

(13) Zhou, C.; Kong, J.; Dai, H. Phys. Rev. Lett. 2000, 84, 5604.

(14) Tans, S. J.; Verscheuren, A. R. M.; Dekker, C. Nature (London) 1998, 393, 49. Bachtold, A.; Hadley, P.; Nakanishi, T.; Dekker, C. Science 2001, 294, 1317.

(15) Soh, H. T.; Quate, C.; Morpurgo, A. F.; Marcus, C. M.; Kong, J.; Dai, H. Appl. Phys. Lett. 1999, 75, 627. Zhou, C.; Kong, J.; Dai, H. Appl. Phys. Lett. 2000, 76, 1597.

(16) Martel, R.; Derycke, V.; Lavoie, C.; Appenzeller, J.; Chan, K. K.; Tersoff, J.; Avouris, Ph. Phys. Rev. Lett. 2001, 87, 256805.

(17) Bagci, V. M. K.; Gülseren, O.; Yildirim, T.; Gedik, Z.; Ciraci, S. Phys. Rev. B 2002, 66, 045409.

(18) Kulik, I. O.; Hakioglu, T.; Barone, A. Eur. Phys. J. B 2002, 30, 219.

(19) Zhang, X. X.; Wen, G. H.; Huang, S.; Dai, L.; Gao, R.; Wang, Z. L. J. Magn. Magn. Mater. 2001, 231, L9. Satishkumar, B. C.; Govindaraj, A.; Vanitha, P. V.; Raychaudhuri, A. K.; Rao, C. N. R. Chem. Phys. Lett. 2002, 362, 301.

(20) Yildirim, T.; Gülseren, O.; Ciraci, S. Phys. Rev. B 2001, 64, 075404. Gülseren, O.; Yildirim, T.; Ciraci, S. Phys. Rev. B 2002, 66, 121401.

(21) Dag, S.; Gülseren, O.; Yildirim, T.; Ciraci, S. Phys. Rev. B 2003, 67,165424

(22) Durgun, E.; Dag, S.; Bagci, V. M. K.; Gülseren, O.; Yildirim, T.; Ciraci, S. Phys. Rev. B 2003, 67, R201401.

(23) Perdew, J. P.; Chevary, J. A.; Vosko, S. H.; Jackson, K. A.; Pederson, M. R.; Singh, D. J.; Fiolhois, C. Phys. Rev. B 1992, 46, 6671.

(24) Gülseren, O.; Yildirim, T.; Ciraci, S. Phys. Rev. Lett. 2001, 87 116802

(25) Gülseren, O; Yildirim, T.; Ciraci, S. Phys. Rev, B 2002, 65, 155410. Gülseren, O.; Yildirim, T.; Ciraci, S.; Kilic, C. Phys. Rev. B 2002, 65, 153405 .

(26) Vanderbilt, D. Phys. Rev. B 1990, 41, 7892.

(27) Monkhorst, H. J.; Pack, J. D. Phys. Rev. B 1976, 13, 5188.

(28) Lifshitz, E. M. Zh. Eksp. Teor. Fiz. 1956, 29, 94; [Lifshitz, E. M. Sov. Phys. JETP 1956, 2, 73].

(29) Halgren, T. A. J. Am. Chem. Soc. 1992, 114, 7827.

(30) Moore, C. E. Atomic Energy Levels; U.S. National Bureau of Standards, Washington, D.C., 1971.

(31) Friedel, J. In The Physics of Metals; Ziman, J. M., Ed.; Cambridge University Press: New York, 1969. Pettifor, D. G. In Solid State Physics, Ehrenreich, H., Turnbull, D., Eds.; Academic Press: New York, 1987; Vol. 40, p 43.

(32) Blase, X. Benedict, L. X.; Shirley, E. L.; Louie, S. G. Phys. Rev. Lett. 1994, 72, 1878 .

(33) Bairle, R. J.; Fagan, S. B.; Mota, R.; Silva, A. J. R.; Fazzio, A. Phys. Rev. B 2001, 64, 085413

(34) Delaney, P.; Choi, H. J.; Ihm, J.; Louie, S. G.; Cohen, M. L. Nature (London) 1998, 391, 466. 